\title{
Supporting information : Generation of spin defects in hexagonal boron nitride
}

\author{
Mehran Kianinia,,${ }^{1 *}$ Simon White, ${ }^{1}$ Johannes E. Fröch, ${ }^{1}$, Carlo Bradac, ${ }^{1}$ and Igor Aharonovich. ${ }^{1,2, *}$ \\ ${ }^{1}$ School of Mathematical and Physical Sciences, University of Technology Sydney, Ultimo, New \\ South Wales, 2007, Australia \\ 2 ARC Centre of Excellence for Transformative Meta-Optical Systems, Faculty of Science, \\ University of Technology Sydney, Ultimo, New South Wales, 2007, Australia \\ *mehran.kianinia@uts.edu.au,
}

\section{Methods}

Ion implantation: we used a ThermoFisher Helios G4 PFIB for ion implantation. For all irradiations the beam energy was set to $30 \mathrm{keV}$. The resolution is stated by the manufacturer to be on the order of $20 \mathrm{~nm}$. The ion beam current was measured using an in-column faraday cup, as 8 $\mathrm{pA}, 20 \mathrm{pA}, 13 \mathrm{pA}$, for the Xe, Ar, and N, respectively. The fluence was calculated as the product of the processing time multiplied by the beam current divided by the elementary charge constant and the irradiated area. Box irradiations were performed using a serpentine scan of the focused ion beam in a square with $4 \mu \mathrm{m}$ sides. The fluence for particular boxes was controlled by setting the number of passes. In each individual pass the dwell time (irradiation time) per spot was set to 1 $\mu \mathrm{s}$. The point pitch (distance between individual spots) was set to $30 \mathrm{~nm}$. The ion beam current was measured directly before the irradiation using an in-column faraday cup, which was 8 pA, 20 $\mathrm{pA}, 13 \mathrm{pA}$, for the Xe, Ar, and N, respectively.

For SRIM calculations, an amorphous volume was assumed with a composition of $\mathrm{B}$ and $\mathrm{N}$ with a $1: 1$ ratio and a density of $2.1 \mathrm{~g} / \mathrm{cm}^{3}$. In calculations the primary ion (Xe, Ar or $\mathrm{N}$ ) was set to an energy of $30 \mathrm{keV}$. Calculations were done using full damage cascades. For the calculations 5,000 ions or more were used. We note that a higher number of ions did not seem to change the statistics of the created vacancies.

Photoluminescnece measurement. A home-built confocal scanning setup was used to characterize the samples. We used a closed-looped piezo stage to scan the samples. A continuous wave 532$\mathrm{nm}$ laser (Laser quantum GEM) was focused into a high numerical aperture (0.9 NA) air objective with power of $1 \mathrm{~mW}$, measured at the back aperture of the objective. The emission was collected in reflection from the same spot. Collection was focused into the core of a 62.5 um multimode fiber which provides a $\mathrm{x}-\mathrm{y}$ spatial resolution of $\sim 500 \mathrm{~nm}$. The collection was either directed into spectrometer (Princeton instruments, with resolution of $0.2 \mathrm{~nm}$ ) or an avalanche photodiode (Excelitas technologies). All spectroscopic measurements were performed with the same excitation power and collected for 30 seconds. For autocorrelation measurement we used a Hanbury-Brown and Twiss interferometer equipped with a high resolution time correlator (Picoquant Picoharp 300, 4ps). For ODMR measurement a copper wire with a diameter of $30 \mu \mathrm{m}$ was placed near the sample. Microwave signal from a generator (Anapico aspin) was amplified (minicircuits, ZHL-16W-43-S+) and directed through the wire. Microwave was swept between 3 to $4 \mathrm{GHz}$ and the difference in PL signal was detected for when microwave was on and off with integration time of 2 seconds. 
(a)

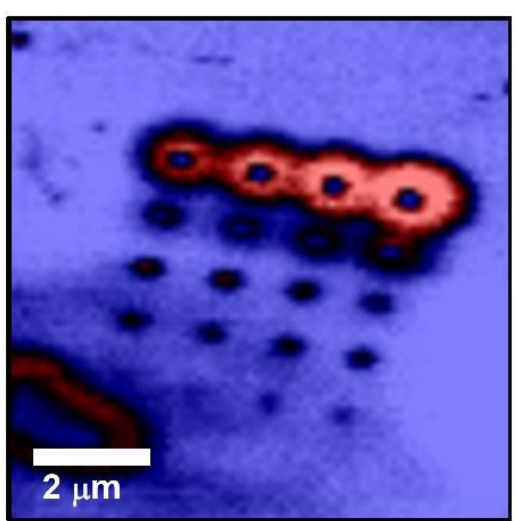

(b)

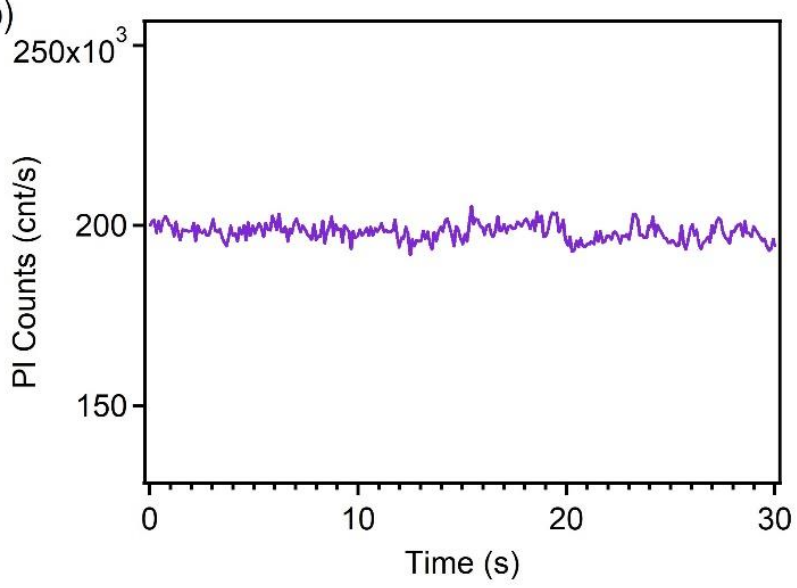

Figure SI1. a) Confocal scan of the point-like pattern. The Xe ion fluence is increased from bottom left to top right. The damage from high fluence is visible on the top two rows. b) Photostability analysis of boron-vacancy defects under $12 \mathrm{~mW}$ of excitation over the period of $30 \mathrm{~s}$. 